TAO, Vol. 14, No. 4, 505-514, December 2003

NOTES AND CORRESPONDENCE

\title{
A Simple Atmospheric Correction Model for ROCSAT-2 RSI Data
}

\author{
Chien-Hui Liu ${ }^{1}$ * \\ (Manuscript received 16 May 2003, in final form 16 October 2003)
}

\begin{abstract}
A simple atmospheric correction model (SACM) for ROCSAT-2 RSI data is presented. Gaseous transmission and Rayleigh optical depth are simplified as analytic functions. Rayleigh and aerosol scattering are determined using lookup tables. Results indicate that SACM not only quite accurately reproduces top-of-atmosphere reflectance computed by the $6 \mathrm{~S}$ model (Vermote et al. 1997), but also does so faster than 6S. The results of the inverse application of SACM also indicate that the error of surface reflectance can be greatly reduced even in hazy sky if the adjacency effect is corrected. Future studies and possible improvements are also highlighted.
\end{abstract}

(Key words: Atmospheric correction model, ROCSAT-2, Reflectance, Adjacency effect)

\section{INTRODUCTION}

The ROCSAT-2 satellite will be launched in the end of 2003 to image over Taiwan and the surrounding area daily for various applications, such as agriculture, land use and disaster monitoring (Lee et al. 2002). It is in a sun-synchronous orbit (inclination angle $98.99^{\circ}$ ) descending over $\left(120^{\circ} \mathrm{E}, 24^{\circ} \mathrm{N}\right)$ and 9:45 a.m. over the equator. The Remote Sensing Instrument (RSI) on board will provide images for $2 \mathrm{~m}$ ground sampling distance (GSD) in panchromatic band and $8 \mathrm{~m} \mathrm{GSD}$ in four Landsat-like multispectral bands. Figure 1 shows the sensor response functions from band 1 to band 4 of ROCSAT-2 RSI. Their central wavelengths correspond to 0.484 (blue), 0.561 (green), 0.660 (red) and 0.817 (near-infrared) $\mu m$, respectively. The central wavelength $\lambda_{c}$ of every spectral band is determined by

\footnotetext{
${ }^{1}$ Department of Information Management/Geographic Information Management and Research Center, Transworld Institute of Technology, Taiwan

${ }^{*}$ Corresponding author address: Prof. Chien-Hui Liu, Department of Information Management/Geographic Information Management and Research Center, Transworld Institute of Technology, 1221, Jen. S. Rd., Douliu, Yunlin 64045, Taiwan; E-mail: chliu@tit.edu.tw
} 


$$
\lambda_{c}=\int \lambda S(\lambda) E(\lambda) d \lambda / \int S(\lambda) E(\lambda) d \lambda
$$

where $S(\lambda)$ and $E(\lambda)$ are sensor response function and exoatmospheric solar irradiance, respectively (Vermote et al. 1997). The RSI will take four-strip images covering the whole of Taiwan during one pass by viewing in $\pm 45^{\circ}$ for along-track and cross-track. Since satellite sensors receive signals not only by reflection from a target but also by atmospheric scattering and absorption, the atmospheric correction of ROCSAT-2 RSI data is necessary to retrieve the land parameters, such as vegetation indices (VIs), leaf area index (LAI) and land use/land cover change. The process of converting raw data to surface reflectance requires the atmospheric correction model. Hence, it's very important to develop the atmospheric correction model for ROCSAT-2 RSI data.

The atmospheric correction of numerous remotely sensed data requires a fast, accurate, and even operational atmospheric correction model. Many researchers have successfully developed such models using the simplified method and/or lookup table approach. Using the simplified functions for gaseous transmission, atmospheric reflectance, spherical albedo and scattering transmission, top-of-atmosphere (TOA) reflectance can be accurately simulated (at approximately 0.001) (Rahman and Dedieu 1994) a few hundred or even several thousand times faster than $5 \mathrm{~S}$ as developed by Tanre et al. (1990). In fact, some other researchers also developed fast and accurate atmospheric correction algorithms using various radiative transfer codes, such as the 5S code by Zagolski and Gastellu-Etchegorry (1995) and the MODTRAN code (Berk et al. 1989) by Richter (1997). The accuracy and limits on the developed models are confined by not only the radiative transfer code itself but also the way researchers implement the code. For example, Liang et al. (1997) determined lookup tables only at discrete wavelengths, whereas Richter (1997) integrated the atmospheric functions with the sensor spectral response function. Some models do not correct the adjacency effect on high resolution images such as Landsat TM images (Liang et al. 1997). Although ATCOR3 (Richter
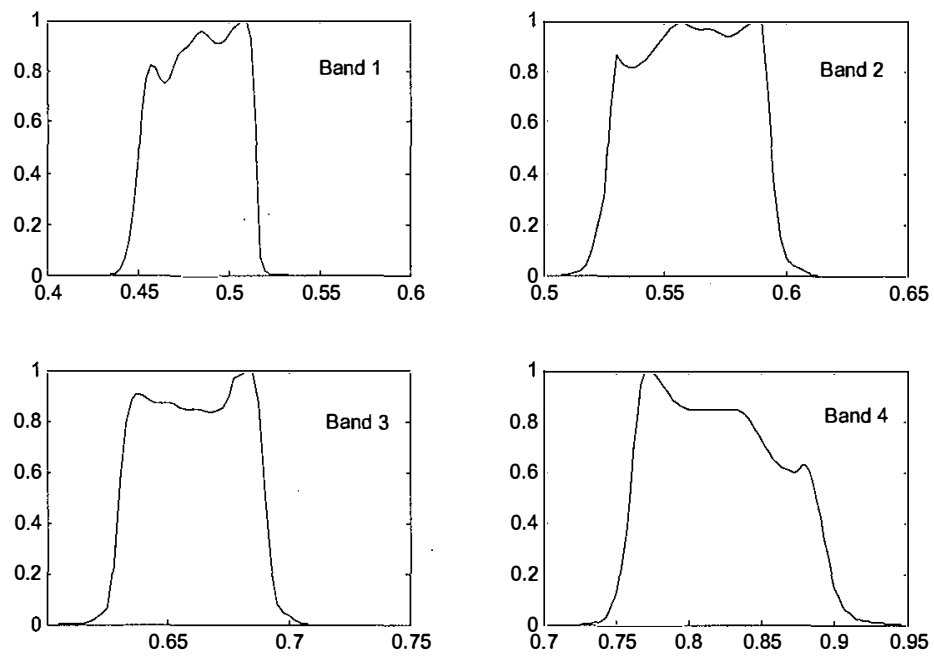

Fig. 1. Sensor response functions from band 1 to band 4 of ROCSAT-2 RSI. 
1997) successfully demonstrate an ability to correct both atmospheric and topographic effects on Landsat TM and SPOT HRV images over a wide range of atmospheric conditions, the azimuthal dependence of atmospheric functions is simplified (relative azimuthal angles of 30 and 150 degrees) to keep down the size of the lookup table for SPOT images. The altitudedependence of atmospheric functions is also usually neglected (Rahman and Dedieu 1994). Consequently, retrieval of surface reflectance may not be accurate, especially for high resolution ROCSAT-2 RSI data over the mountainous area of Taiwan.

In this paper, a simple atmospheric correction method (SACM) for ROCSAT-2 RSI data is developed. The 6S radiative transfer code (Vermote et al. 1997) is used, because it is accurate and has been successfully used in the development of an atmospheric correction model for EOS MODIS (Vermote et al. 2002) data. Since SACM utilizes simplified functions for gaseous transmission and Rayleigh optical depth and lookup tables for atmospheric scattering, it can rapidly (several hundred times faster than $6 \mathrm{~S}$ ) and accurately ( 0.001) reproduce TOA reflectance. When SACM is used in the inverse mode, it can also convert the TOA reflectance to surface reflectance with correction of adjacency effect.

\section{SIMPLE ATMOSPHERIC CORRECTION METHOD (SACM)}

SACM is based on the $6 \mathrm{~S}$ radiative transfer model, whose outputs are considered to determine simplified functions for gaseous transmission and Rayleigh optical depth, and lookup tables for Rayleigh and aerosol scattering. It considers various altitudes (but not on sloped terrain) and can correct the adjacency effect. The approach somewhat follows the methodology of Vermote et al. (2002). All of the transmission functions and scattering lookup tables of the various gases and aerosol are computed by $6 \mathrm{~S}$ and spectrally integrated with the sensor response functions for the four bands of ROCSAT-2 RSI. For simplicity, only the continental aerosol model is considered here.

\section{RESULTS AND DISCUSSIONS}

Several experiments have been conducted to validate SACM. These compare gaseous transmission, scattering terms and TOA reflectance obtained by SACM and 6S. The experiments also show the advantage of SACM over $6 \mathrm{~S}$ in terms of execution speed, as well as the correction of the adjacency effect.

The performance of SACM is evaluated in terms of maximum relative error (MRE) and root mean square error with respect to $6 \mathrm{~S}$. The MRE is defined as $100\left|X_{i}-X_{6 S}\right| / X_{6 S}$ and rmse $=\sqrt{\left(X_{i}-X_{6 S}\right)^{2} /(N-1)}$, with $X_{i}$ derived by SACM and $X_{6 S}$ according to 6 S. Such validation is analogous to that employed by Rahman and Dedieu (1994).

\subsection{Gaseous Transmissions and Rayleigh Optical Depth}

Table 1 presents the regressed coefficients of the simplified functions of ozone, water 
vapor, oxygen transmissions and Rayleigh optical depth for various bands of ROCSAT-2 RSI. The corresponding MRE and rmse values are also calculated. 6S is used at various ranges to evaluate the fitness of the simplified functions. Tropical atmosphere is used. Both solar zenith angle $\theta_{s}$ and viewing zenith angle $\theta_{v}$ are from $0^{\circ}$ to $60^{\circ}$, and therefore the air mass varies from 2 to 4 . Ozone content in sea level $U_{O_{3}}(0)$ is varied from 0.1 to $0.6 \mathrm{~cm}$-atm to evaluate the performance of ozone transmission $T_{g_{O_{3}}}$. The MRE values are $0.0000,0.0001,0$. $0001,0.0000$ and $0.0004 \%$ from band 1 to band 5 and the corresponding rmse values are all the same as those of MRE. These results indicate that the ozone transmission calculated by SACM agrees very well with that of $6 \mathrm{~S}$ for all bands.

The simulated water vapor transmission $T_{g_{\mathrm{H}_{2}} \mathrm{O}}$ values obtained with water vapor content in sea level $U_{\mathrm{H}_{2} \mathrm{O}}(0)$ from 1.0 to $10.0 \mathrm{~g} \mathrm{~cm}^{-2}$ are also compared with those of $6 \mathrm{~S}$. The MRE values are all 0.0000 for all bands except 0.0001 for band 4 , and the corresponding rmse values are all 0.0000 for all bands. The MRE and rmse values of $T_{g_{H_{2} O}}$ with $U_{H_{2} O}(0) / 2$ are of the same order with those of $T_{\mathrm{H}_{2} \mathrm{O}}$ values obtained with $U_{\mathrm{H}_{2} \mathrm{O}}(0)$, although the MRE and rmse values exceed those of transmission with full water vapor content for band 2, band 4 and band 5. These results demonstrate a very good agreement between the water vapor transmission determined by SACM and that by $6 \mathrm{~S}$.

The absorption of gases other than ozone and water vapor, including carbon dioxide, oxygen and methane, are relatively unimportant in the spectral bands of ROCSAT-2 RSI. The oxygen transmission as a function of the product of airmass and the ratio of pressure to sea level pressure is determined for band 3, band 4 and band 5. Airmass varies from 2 to 4 . Oxygen content is equal to one since the concentration is effectively constant (Rahman and Dedieu 1994). Altitude ranges from 0 to $3.2 \mathrm{~km}$. The MRE and rmse values are 0.0000 for band 3, band 4 and band 5, which indicates the good agreement between SACM and 6S for oxygen transmission computation.

Rayleigh optical depth $\tau_{R}$ as a function of altitude for the tropical atmospheric model is also determined. Altitude varies from 0 to $7 \mathrm{~km}$. Although the MRE values are all $0.0117 \%$, the rmse values are only $0.0008,0.0004,0.0002,0.0001$ and 0.0003 from band 1 to band 5, which depicts $\tau_{R}$ computed by SACM agrees very well with that by $6 \mathrm{~S}$.

The above discussion shows that the gaseous transmission and Rayleigh optical depth computed by SACM agree very closely with those by $6 \mathrm{~S}$.

\subsection{Lookup Tables for Rayleigh and Aerosol Scattering}

To assess the performance of the lookup tables for atmospheric spherical albedo $S$, diffuse transmittance of molecules $t_{d}^{R}(\mu)$, diffuse transmittance of aerosol $t_{d}^{A}(\mu)$, environment function $F(r)$, atmospheric reflectance of Rayleigh $\rho_{R}$ and atmospheric reflectance of aerosol $\rho_{A}$, these scattering components are computed at two altitudes $(0$ and $4 \mathrm{~km})$, three solar zenith angles $\theta_{s}\left(0^{\circ}\right.$ to $60^{\circ}$, every $\left.30^{\circ}\right), 4$ viewing zenith angles $\theta_{v}\left(0^{\circ}\right.$ to $45^{\circ}$, every $\left.15^{\circ}\right), 4$ relative azimuthal angles $\phi\left(0^{\circ}\right.$ to $180^{\circ}$, every $\left.60^{\circ}\right)$, and 4 aerosol optical depths $(550 \mathrm{~nm})(0.05$ to 0 . 8 , every 0.25 ). The aerosol model is continental type. It should be noticed that these scattering components are computed in conditions different from those in the lookup tables. Therefore, the errors of Rayleigh and aerosol scattering are due to interpolation using these lookup tables 
Table 1. The regressed coefficients of the simplified functions of ozone, water vapor, oxygen transmissions and Rayleigh optical depth for various bands of ROCSAT-2 RSI. Air mass ranges from 2 to $4 . U_{O_{3}}(0)$ ranges from 0.1 to $0.6 \mathrm{~cm}$-atm. $U_{\mathrm{H}_{2} \mathrm{O}}(0)$ ranges from 1.0 to $10.0 \mathrm{~g} \mathrm{~cm}^{-2}$. For other gases, altitude ranges from 0 to $3.2 \mathrm{~km}$. Tropical atmospheric is used.

\begin{tabular}{ccccccc}
\hline & & Band 1 & Band 2 & Band 3 & Band 4 & Band 5 \\
\hline $\mathrm{O}_{3}$ & $\mathrm{a}$ & 0.0190 & 0.0948 & 0.0565 & 0.0007 & 0.0427 \\
\hline & $\mathrm{a}$ & -26.6444 & -5.9611 & -6.0626 & -3.8764 & -4.5883 \\
$\mathrm{H}_{2} \mathrm{O}^{*}$ & $\mathrm{~b}$ & 0.5488 & 1.0104 & 1.0195 & 0.7054 & 0.7794 \\
& $\mathrm{c}$ & -0.0579 & -0.0589 & -0.0508 & -0.0332 & -0.0405 \\
\hline & $\mathrm{a}$ & -26.6444 & -6.6655 & -6.7634 & -4.4027 & -5.1581 \\
$\mathrm{H}_{2} \mathrm{O}^{\#}$ & $\mathrm{~b}$ & 0.5488 & 1.0742 & 1.0676 & 0.7676 & 0.8432 \\
& $\mathrm{c}$ & -0.0579 & -0.0557 & -0.0468 & -0.0361 & -0.0419 \\
\hline & $\mathrm{a}$ & - & - & 0.0106 & 0.0219 & 0.0097 \\
$\mathrm{O}_{2}$ & $\mathrm{~b}$ & - & - & 0.4699 & 0.3471 & 0.3628 \\
\hline & $\tau_{R}(0)$ & 0.1700 & 0.0925 & 0.0474 & 0.0203 & 0.06923 \\
& $H_{\tau_{R}}$ & 7.8357 & 7.8357 & 7.8357 & 7.8357 & 7.8357 \\
\hline
\end{tabular}

*: for $\mathrm{U}_{\mathrm{H}_{2} \mathrm{O}}$; : for $\mathrm{U}_{\mathrm{H}_{2} \mathrm{O}} / 2$.

as compared with the values calculated by $6 \mathrm{~S}$ under the same afore-mentioned conditions.

The MRE values and the rmse values of atmospheric scattering terms are computed. The rmse values of $t_{d}^{R}(\mu)$ and $\rho_{R}$ are less than 0.0002 and 0.0003 from band 1 to band 5, although their MRE values are all greater than $1.0 \%$. The rmse values of $t_{d}^{A}(\mu)$ are $0.0040,0.0033$, $0.0026,0.0015$ and 0.0015 from band 1 to band 5 , which exceed an order of magnitude higher than those of $t_{d}^{R}(\mu)$. The MRE values of $t_{d}^{A}(\mu)$ are also larger than those of $t_{d}^{R}(\mu)$. One would have noticed that the rmse values of $t_{d}^{A}(\mu)$ are the largest compared with those of other scattering terms. However, the overall errors of TOA reflectance $\rho_{\text {TOA }}$ are around 0.001 for all bands, as one can see in the following discussions. Even though the MRE values of $\rho_{A}$ can reach $30.43,10.86,7.908,7.454$ and 9.564 from band 1 to band 5 , their corresponding rmse values are only $0.0008,0.0007,0.0006,0.0004$ and 0.0006 , respectively. The MRE values of 
$S$ are all less than $1.0 \%$ for all bands except band 4 , and the rmse values are all around 0.0007 . The MRE values of $F(r)$ are all less than $1.0 \%$ for all bands, and the rmse values are all very low (0.0000).

The rmse values of all scattering components are relatively low, and thus lead to the satisfactorily accurate simulation of TOA reflectance by SACM, as described in the following section.

\subsection{The Accuracy Assessment of the Simulation of TOA Reflectances by SACM}

Figure 2 compares TOA reflectances $\rho_{T O A}$ simulated by SACM and $6 \mathrm{~S}$ for band 1, band 3 , band 4 and band 5 . Wide ranges of parameters are set as follows to determine the accuracy of SACM: altitude $z$ varies from 0 to $4.0 \mathrm{~km} ; \theta_{s}$ and $\theta_{v}$ vary from $0^{\circ}$ to $60^{\circ}$ and from $0^{\circ}$ to $45^{\circ}$ respectively; $\phi$ varies from $0^{\circ}$ to $180^{\circ} ; U_{\mathrm{O}_{3}}(0)$ and $U_{\mathrm{H}_{2} \mathrm{O}}(0)$ vary from 0.1 to $0.7 \mathrm{~cm}$-atm and from 0.5 to $7.0 \mathrm{~g} \mathrm{~cm}^{-2}$, respectively; AOD varies from 0.05 to 0.8 ; the aerosol model is of the continental type, and three target reflectance $\rho_{s}$ and background reflectance $\rho_{b}$ values $(0.05$, 0.25 and 0.45 ) are used. The MRE values of $\rho_{T O A}$ are 2.413, 2.416, 2.471, 2.376 and $3.072 \%$ from band 1 to band 5 , and the corresponding rmse values are less than $0.001(0.0008,0.0007$, 0.0006 and 0.0006 from band 1 to band 5, respectively) except for band 5 (0.0016). From the
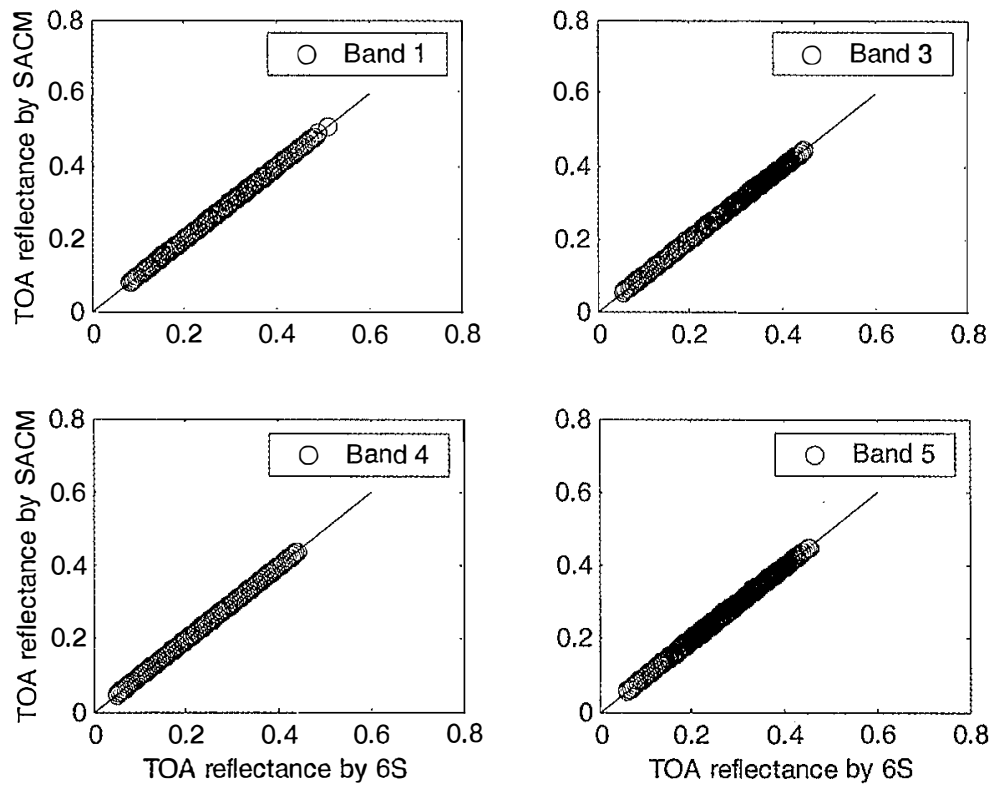

Fig. 2. Comparison between the TOA reflectances as determined by $6 \mathrm{~S}$ and SACM for various bands of ROCSAT-2 RSI. $\theta_{s}$ ranges from $0^{\circ}$ to $60^{\circ}$, $\theta_{v}$ from $0^{\circ}$ to $45^{\circ}, \phi$ from $0^{\circ}$ to $180^{\circ}$ and AOD from 0.05 to $0.8 . U_{O_{3}}(0)$ and $U_{H_{2} O}(0)$ vary from 0.1 to $0.7 \mathrm{~cm}$-atm and from 0.5 to $7.0 \mathrm{~g} \mathrm{~cm}^{-2}$. Aerosol optical depth varies from 0.05 to 0.8 . The altitude is from 0 to 4 . $0 \mathrm{~km}$. Surface and background reflectances are $0.05,0.25$, and 0.45 . 
above discussions, therefore, SACM can mimic $6 \mathrm{~S}$ with sufficient accuracy.

Table 2 compares the CPU time required for 1024 runs of TOA reflectance calculation for various bands using $6 \mathrm{~S}$ and SACM on a personal computer (Pentium 4 with $1.7 \mathrm{GHz} \mathrm{CPU}$ ). The computational time required for $6 \mathrm{~S}$ varies significantly with spectral bands and is almost constant for SACM. For band 1, SACM is 614 times faster than $6 \mathrm{~S}$ and for band 4 it is 425 times more rapid. For panchromatic band (band 5), SACM is 1191 times more rapid than 6S. One can see that as the bandwidth increases the computational time for $6 \mathrm{~S}$ increases because of the necessity of the spectral integration. Hence, SACM is also appropriate for correcting the atmospheric effects of ROCSAT-2 RSI data.

\subsection{Determination of Surface Reflectance with Correction for the Adjacency Effect}

Figure 3 presents the example of simulation of TOA reflectance and atmospherically corrected surface reflectance with/without correction of adjacency effect by SACM for spectral bands of ROCSAT-2 RSI. Vegetation surface (target) has assumed to be surrounded by soil surface (background). Both vegetation and soil reflective spectra refer to spectral reflectances for different dry green biomass levels for alfalfa canopy (Fig. 10 of Deering (1989)). $\theta_{s}, \theta_{v}$ and $\phi$ are $60^{\circ}, 45^{\circ}$ and $180^{\circ}$, respectively. $U_{\mathrm{O}_{3}}(0)$ and $U_{\mathrm{H}_{2} \mathrm{O}}(0)$ are $0.3 \mathrm{~cm}$-atm and 4.0 $\mathrm{g} \mathrm{cm}^{-2}$, respectively. Visibility is $5 \mathrm{~km}$. The altitude is in sea level. As one can see in Fig. 3, TOA reflectances are much higher than surface reflectances in blue, green and red bands of ROCSAT-2 RSI. It's because of the stronger atmospheric scattering effect for the short wavelengths. TOA reflectance in near-infrared band is lower than the surface reflectance mainly due to the aerosol and water vapor absorption. Therefore, this emphasizes again the necessity of the atmospheric correction of remotely sensed signal. When the atmospheric effect has been corrected without considering the adjacency effect, i.e. uniform surface assumption, the retrieved vegetation spectral reflectances are much closer to the surface

Table 2. Comparison of CPU time (seconds) required for 1024 iterations of TOA reflectance calculation for different bands of ROCSAT-2 RSI using $6 \mathrm{~S}$ and SACM on a personal computer (Pentium 4 with $1.7 \mathrm{GHz}$ CPU).

\begin{tabular}{ccc}
\hline Band & $6 \mathrm{~S}$ & SACM \\
\hline 1 & 1842 & 3 \\
2 & 1818 & 3 \\
3 & 1751 & 3 \\
4 & 1275 & 3 \\
5 & 3574 & 3 \\
\hline
\end{tabular}


reflectances, although there still exist some deviations. Such deviations, which are due to the atmospheric forward scattering from the background (soil), can be reduced if the adjacency effect is corrected.

This experiment reveals the necessity of correcting for the adjacency effect on high- resolution ROCSAT-2 RSI data.

\section{CONCLUSIONS}

The main contributions of this paper are the development of the simple atmospheric correction model (SACM) suited to ROCSAT-2 RSI data. Compared with 6S, SACM is reasonably accurate and much faster, and thus can be applied to satellite receiving stations for the atmospheric correction of ROCSAT-2 RSI data in near real time.

It should be emphasized that an accurate atmospheric correction model may not correct the atmospheric effects well on numerous remotely sensed data without knowledge of the

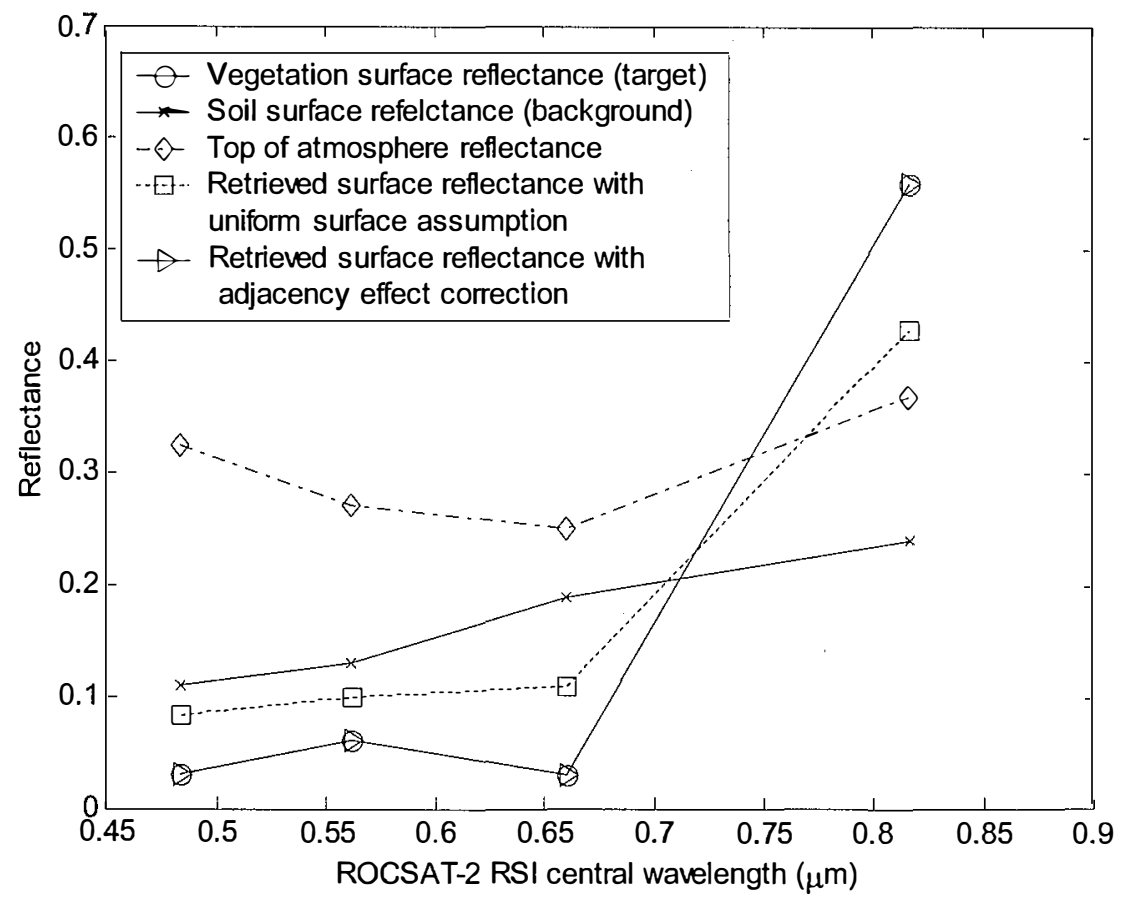

Fig. 3. Example of simulation of top of atmosphere reflectance and atmospherically corrected surface reflectance with/without correction of adjacency effect by SACM for spectral bands of ROCSAT-2 RSI. Vegetation surface (target) has assumed to be surrounded by soil surface (background). $\theta_{s}, \theta_{v}$ and $\phi$ are $60^{\circ}, 45^{\circ}$ and $180^{\circ}$, respectively. $U_{O_{3}}(0)$ and $U_{H_{2} O}(0)$ are $0.3 \mathrm{~cm}$-atm and $4.0 \mathrm{~g} \mathrm{~cm}^{-2}$, respectively. Visibility is $5 \mathrm{~km}$. The altitude is in sea level. 
atmospheric parameters (Rahman and Dedieu 1994), particularly the aerosol concentration, due to its spatial and temporal variation. To be operational, the only method to obtain the aerosol concentration of ROCSAT RSI data is to retrieve the AOD from the image itself. Although the dark target (DT) approach has been operationally applied to Landsat TM (Liang et al. 1997) and EOS MODIS data (Vermote et al. 2002), the contrast reduction (CR) based methods, such as "structure method" (Lin et al. 2002) and "dispersion method" (Liu et al. 2002), can be alternative ways to successfully estimate the AOD from the complex terrains for SPOT HRV and NOAA AVHRR data. The major assumptions of the DT approach are the existence and referenced reflectances in visible bands of DT, and those of the CR method are unchanged ground reflectances within multi-temporal data and the difference between the data satisfactorily attributed to the difference of AOD. In considering the four bands of ROCSAT-2 RSI, both methods are suitable. However, further investigation is suggested. Nevertheless, SACM can be used inversely to retrieve AOD by using both approaches, since it is fairly accurate and fast.

Currently, SACM adopts only the continental aerosol model, which is not appropriate for urban pollution, fog or even Asian dust storms, which are very active in spring. Although altitude dependence is considered, SACM does not consider the topographic effects of a sloped surface also. Future work will improve SACM accordingly.

Acknowledgements The author is grateful to Mr. Sz-Yuan Lee at National Space Program Office for his help or the spectral response functions of the ROCSAT-2 RSI. The anonymous reviewers' comments led to significant improvements in the manuscript. This work was supported by the Taiwan National Science Council under grant NSC91-2212-E-265-001.

\section{REFERENCES}

Berk, A., L. S. Bernstein, and D. C. Robertson, 1989: MODTRAN: a moderate resolution model for LOWTRAN 7, GL-TR-89-0122, Geophysics Laboratory, Bedford, MA, U. S. A.

Deering, D. W., 1989: Field measurements of bidirectional reflectance. In: Theory and Applications of Optical Remote Sensing, G. Asrar (Eds.), John Wiley \& Sons, 37-38.

Lee, Y. Y., A. M. Wu, and F. Wu, 2002: An algorithm for geometric correction of high resolution image based on physical modeling. In the $23^{\text {nd }}$ Asian Conference on Remote Sensing, 23-29 November 2002, Nepal.

Liang, S., H. Fallah-Adl, S. Kalluri, J. Jaja, Y. J. Kaufman, and J. R. G. Townshend, 1997: An operational atmospheric correction algorithm for Landsat Thematic Mapper imagery over the land. J. Geophy. Res., 102(D14), 17173-17186.

Lin T. H., A. J. Chen, G. R. Liu, and T. H. Kuo, 2002: Monitoring the atmospheric aerosol optical depth with SPOT data in complex terrain. Int. J. Remote Sens., 23, 647-659.

Liu G. R., T. H. Lin, and T. H. Kuo, 2002: Estimation of aerosol optical depth by applying the optimal distance number to NOAA AVHRR data. Remote Sens. Environ., 81, 247-252.

Rahman, H., and G. Dedieu, 1994: SMAC: a simplified method for the atmospheric correction of satellite measurements in the solar spectrum. Int. J. Remote Sens., 15, 123-143. 
Richter, R., 1997: Correction of atmospheric and topographic effects for high spatial resolution satellite imagery. Int. J. Remote Sens., 18, 1099-1111.

Tanre, D., C. Deroo, P. Duhaut, M. Herman, J.-J. Morcrette., J. Perbos, and P. Y. Deschamps, 1990: Description of a computer code to simulate the satellite signal in the solar spectrum: the 5S code. Int. J. Remote Sens., 11, 659-668.

Vermote, E. F., Nazmi Z. El Saleous, and C. O. Justice, 2002: Atmospheric correction of MODIS data in the visible to middle infrared first results. Remote Sens. Environ., 83, 97-111.

Vermote, E. F., D. Tanre, J. L. Deuze, M. Herman, and J.-J. Morcrette, 1997: Second simulation of the satellite signal in the solar spectrum, 6S: an overview. IEEE Trans. Geosc. Remote Sens., 35(3), 675-686.

Zagolski, F., and J. P. Gastellu-Etchegorry, 1995: Atmospheric corrections of AVIRIS with a procedure based on the inversion of the 5S model. Int. J. Remote Sens., 16, 3115-3146. 